\title{
Reabilitação de dentes afetados pela Hipomineralização Molar-Incisivo (HMI): um relato de caso com 16 meses de acompanhamento
}

\author{
Rehabilitation of teeth affected by Molar-Incisor Hypomineralization \\ $(\mathrm{MIH})$ : a case report with 16 months of follow-up
}

Ivam Freire da Silva Júnior

Carlota Rocha de Oliveira**

Paula da Silva Berwig ${ }^{* * *}$

Lisandrea Rocha Schardosim ${ }^{* * *}$

\section{Resumo}

Objetivo: relatar um caso clínico de uma criança diagnosticada com Hipomineralização Molar-Incisivo (HMI) severa, apresentando a proposta terapêutica e o acompanhamento. Relato de caso: uma paciente do sexo feminino, com 6 anos de idade, foi levada a um serviço odontológico com queixa principal de "dor no dente de baixo (lado direito)". No exame clínico, foram constatadas opacidades demarcadas no esmalte dentário, de coloração variando de branco a tons de marrom, características de hipomineralização, nas superfícies vestibulares dos dentes $12,11,21,32,31$ e 41 e nas oclusais do 16 e 26 , sem perda de estrutura dentária. Nos dentes 36 e 46, observaram-se, além das opacidades demarcadas, fraturas de esmalte pós-eruptivas associadas com lesões ativas de cárie em dentina. Clinicamente e radiograficamente, não havia sinais de comprometimento da saúde pulpar. A criança foi então diagnosticada com HMI. O tratamento consistiu em restauração direta em resina composta dos elementos 36 e 46. Após 16 meses, observou-se que não houve perda de material restaurador em ambas as restaurações. A criança não relatou sensibilidade dentinária e nem sintomatologia dolorosa. Considerações finais: o tratamento proposto para o caso mostrou-se satisfatório durante o período avaliado, pois recuperou a funcionalidade dos dentes afetados pela HMI e não houve mais eventos de sensibilidade e dor.

Palavras-chave: Assistência odontológica. Criança. Desmineralização do dente. Esmalte dentário. Saúde bucal.

\section{Introdução}

Nas últimas décadas, a odontologia tem dado atenção especial às patologias pertinentes aos Defeitos de Desenvolvimento do Esmalte (DDE), principalmente pela expressiva prevalência, inconclusiva etiologia e complexidade do manejo clínico $^{1-3}$. Hipoplasias são definidas como defeitos quantitativos do esmalte, em que a injúria ocorre na fase secretora da formação da matriz do esmalte. Hipomineralizações resultam de um dano causado ao esmalte dentário durante a sua fase de maturação e caracterizam-se por defeitos qualitativos do esmalte dentário ${ }^{4,5}$.

Hipomineralização Molar-Incisivo (HMI) foi a nomenclatura proposta para definir as opacidades no esmalte, de etiologia sistêmica e que afetam de um ou até os quatro primeiros molares permanentes, associadas ou não aos incisivos permanentes ${ }^{6}$. Clinicamente, esses dentes apresentam-se com opacidades demarcadas de coloração branca, amarela ou castanha. $\mathrm{O}$ esmalte é poroso e, por isso, suscetível às fraturas, podendo expor a dentina e contribuir para o desenvolvimento de lesões cariosas ${ }^{6}$. Uma vez que os túbulos dentinários estejam expostos, a sensibilidade dentinária faz parte da rotina do paciente com $\mathrm{HMI}^{6}$. Como consequência da HMI, a hipersensibilidade e o desenvolvimento rápido das lesões cariosas, assim como os tratamentos dentários recorrentes, podem ocasionar efeitos sociais e estéticos negativos na vida cotidiana do paciente ${ }^{7}$.

\footnotetext{
Mestre, Programa de Pós-Graduação em Odontologia, Universidade Federal de Pelotas, Pelotas, Rio Grande do Sul, Brasil.

Cirurgiã-dentista, Faculdade de Odontologia, Universidade Federal de Pelotas, Pelotas, Rio Grande do Sul, Brasil.

Cirurgiã-dentista, Faculdade de Odontologia, Universidade Federal de Pelotas, Pelotas, Rio Grande do Sul, Brasil.

Doutora, Departamento de Odontologia Social e Preventiva, Faculdade de Odontologia, Universidade Federal de Pelotas, Pelotas, Rio Grande do Sul, Brasil.
} 
A etiologia da HMI é inconclusiva, mas se sabe que pode ser o resultado de uma variedade de fatores ambientais que atuam em nível sistêmico, desde fatores presentes no período pré-natal, no perinatal e na infância tenra ${ }^{8}$. Condições médicas da gestante e da criança que acarretam hipocalcemia e hipóxia parecem estar associadas à $\mathrm{HMI}^{8}$. A predisposição genética é uma possibilidade que não é descartada pela literatura ${ }^{8}$. A prevalência global da HMI é de $14,2 \%{ }^{9}$. No Brasil, observa-se uma variação entre $8,8 \%^{10}$ e $40,2 \%{ }^{11}$

São muitas as possibilidades de intervenção disponíveis para o manejo da HMI, variando desde prevenção de cáries e decomposição do esmalte, tratamentos restauradores com diversos materiais, confecção de coroas metálicas, até procedimentos mais invasivos, como a exodontia ${ }^{12,13}$. Para a tomada de decisão, deve-se avaliar individualmente as necessidades do paciente, levando em consideração a gravidade das lesões, a sintomatologia, a idade e a expectativa estética e funcional ${ }^{12}$.

Ainda que haja muitas possibilidades de manejo de molares afetados pela HMI, não há uma consensualidade de qual seria o tratamento ideal para cada condição ${ }^{12}$. O tratamento tem sido um desafio na prática clínica odontológica, seja por questões de sensibilidade, rápido desenvolvimento e progressão de cáries, cooperação limitada de paciente infantil, dificuldade de alcançar um bom efeito anestésico, seja por problemas como a adesividade e a longevidade de materiais restauradores, em virtude da porosidade do tecido dentinário, podendo resultar em fraturas marginais ${ }^{5}$. Comportamentalmente, esses pacientes tendem a apresentar sentimentos como ansiedade e medo do cirurgião-dentista, possivelmente isso é explicado pelo prolongado tratamento ao qual estão expostos ${ }^{2}$. Segundo Jälevik e Klingberg $^{2}$ (2002), as crianças com HMI são submetidas dez vezes mais a tratamentos do que aquelas sem esta condição.

Por ser uma condição recentemente descrita na literatura, há uma escassez de dados no que tange a critérios para tomada de decisão de tratamento e eleição de materiais restauradores em pacientes infantis, bem como de um melhor detalhamento do transoperatório e do acompanhamento longitudinal do tratamento proposto.

O objetivo deste trabalho é relatar um caso clínico de uma criança diagnosticada com HMI severa, apresentando a proposta terapêutica e o acompanhamento.

\section{Relato de caso}

Uma paciente do sexo feminino, com 6 anos de idade, foi atendida na clínica infantil da Faculdade de Odontologia da Universidade Federal de Pelotas com queixa principal de "dor no dente de baixo (lado direito)", além de relatar sensibilidade dentinária a estímulos térmicos e à ingestão de alimentos açucarados.

Por meio da anamnese realizada com a mãe, não houve nenhuma consideração pertinente ao período pré-natal. A criança nasceu a termo, com parto cesariano, sem nenhuma complicação e com ótimas condições, além de um peso adequado para a idade gestacional $(3,260 \mathrm{Kg})$. A mãe relatou que, durante os três primeiros anos de vida, a criança apresentou repetitivos quadros de infecção urinária, sempre acompanhada de febre, e que, por esse motivo, houve uso frequente de antibióticos. No momento da anamnese, não foi relatado nenhum comprometimento sistêmico e nem uso de medicamentos. Foi realizado um diário alimentar, que permitiu inferir que a criança apresentava uma dieta rica em açúcar.

No exame clínico, foram constatadas opacidades demarcadas no esmalte dentário, de coloração variando de branco a tons de marrom, características de hipomineralização, nas superfícies vestibulares dos dentes 12, 11, 21, 32, 31 e 41 (Figura 1) e nas oclusais do 16 e 26 (Figura 2), sem perda de estrutura dentária. Nos dentes 36 e 46, observaram-se, além das opacidades demarcadas nas faces vestibulares e oclusais, fraturas de esmalte pós-eruptivas associadas com lesões ativas de cárie em dentina (Figura 3). Foi observada, ainda, opacidade demarcada na face vestibular do 85 (Figura 3). Os elementos 54 e 75 apresentaram lesão de cárie ativa em dentina, e o elemento 65 apresentou uma microcavidade de cárie em esmalte. Clinicamente, nenhum dente apresentou fístula ou qualquer outro sinal característico de envolvimento pulpar. Radiograficamente, foi observado que o elemento 46 apresentava lesão de cárie em metade interna de dentina, mas sem sinais de comprometimento da saúde pulpar, o elemento 75 apresentava extensa área radiolúcida na região inter-radicular e menos de dois terços de raiz.

Figura 1 - Vista frontal revelando opacidades demarcadas nos incisivos superiores e inferiores

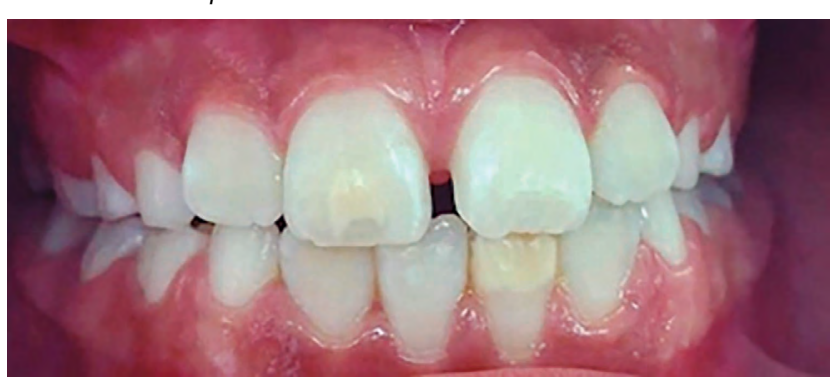

Fonte: autores. 
Figura 2 - Vista oclusal superior revelando opacidades demarcadas nos elementos 16 e 26

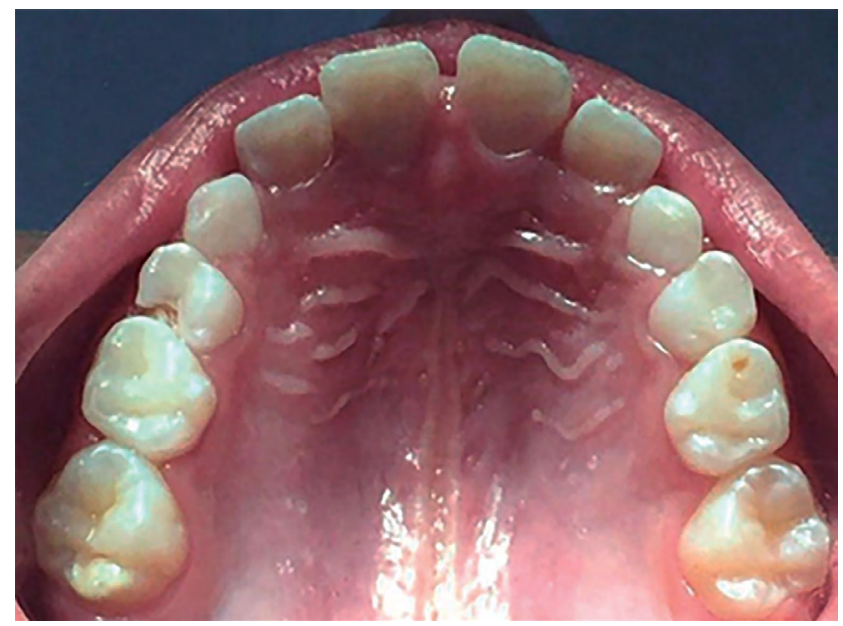

Fonte: autores.

Figura 3 - Vista oclusal inferior revelando opacidades demarcadas e fratura pós-eruptiva do esmalte dos elementos 36 e 46 (A) - Vista vestibular do elemento 36 , revelando o defeito de esmalte (B) - Vista vestibular dos elementos 46 (perda de estrutura) e 85 (opacidades de esmalte) (C)

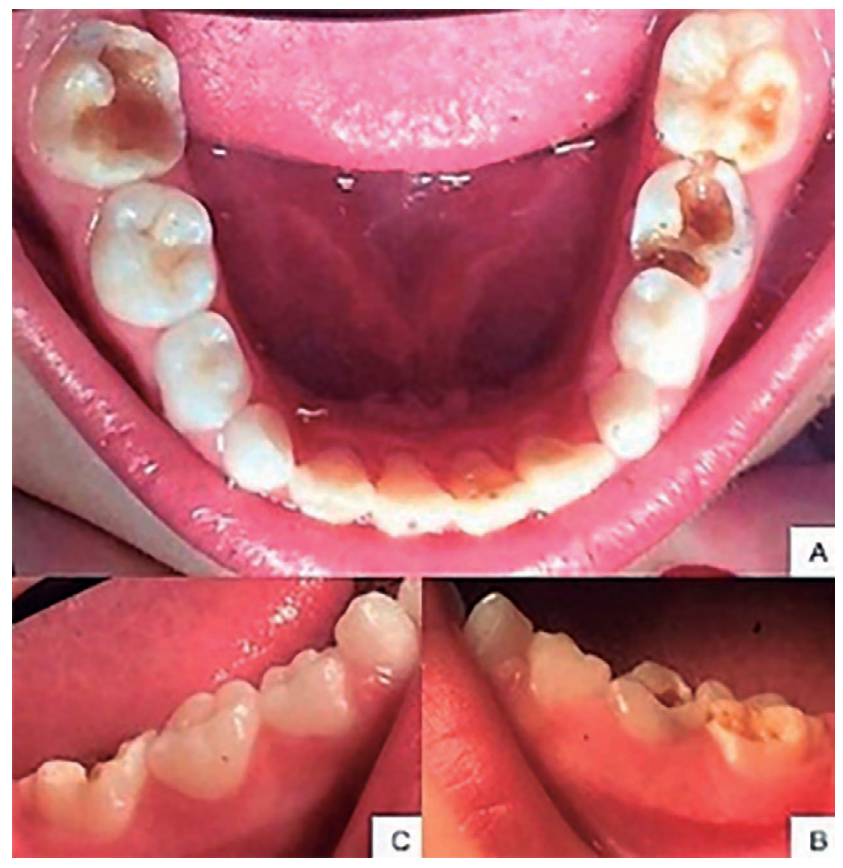

Fonte: autores.

A criança foi então diagnosticada com HMI, além de apresentar experiência e atividade de cárie. O plano de tratamento envolveu instrução e motivação da higiene bucal e da dieta da criança, reabilitação estética e funcional com resina composta nos elementos 36, 46 e 54, exodontia do 75 e, devido a esta perda precoce, foi planejado também o manejo do espaço com aparelho ortodôntico do tipo banda-alça. Tendo em vista que a criança não relatou queixa estética das opacidades localizadas nos incisivos superiores e inferiores, optou-se pela proservação desses elementos.

Do ponto de vista ético, a execução do tratamento e a publicação do caso foram autorizadas pela mãe e pela própria criança, as quais assinaram o termo de consentimento livre e esclarecido.

Primeiramente, foi procedida à restauração do dente 46 , uma vez que, além de ser a principal queixa da paciente, era o dente que apresentava maior perda de estrutura e, por conseguinte, uma maior superfície de exposição dentinária. Foi realizada sob anestesia de bloqueio do nervo alveolar inferior e isolamento absoluto. A remoção do tecido cariado e do esmalte alterado foi feita com alta rotação e com broca carbide, posteriormente, foi realizado um forramento restrito à parede pulpar com cimento à base de ionômero de vidro (CIV) de presa química com o Maxxion R A3 Universal (FGM ${ }^{\circledR}$, Santa Catarina, Brasil). Após o condicionamento ácido com ácido fosfórico a 37\%, procedeu-se à aplicação do sistema adesivo Adper Single Bond $2^{\mathrm{TM}}\left(3 \mathrm{M}\right.$ Espe ${ }^{\circledR}$, São Paulo, Brasil). Sendo assim, por meio da técnica incremental, foi realizada restauração em resina composta utilizando a Z100 ${ }^{\mathrm{TM}}$ (3M Espe ${ }^{\circledR}$, São Paulo, Brasil). Cada incremento foi polimerizado por 20 segundos, sendo que a polimerização final foi de 40 segundos. Após a remoção do lençol de borracha, foram feitos ajustes oclusais e procedimentos de acabamento. Depois de 15 dias, foi realizado o polimento da restauração.

$\mathrm{Na}$ consulta subsequente, foi realizada restauração do elemento 36 nas mesmas condições do que fora feito no elemento 46, exceto pelo fato de não ter sido utilizado forramento com CIV, uma vez que a cavidade era rasa e com pequena área de restauração. Neste dente foi utilizado adesivo autocondicionante Ambar (FGM ${ }^{\circledR}$, Joinville, Brasil), sendo, por isso, realizado apenas condicionamento ácido seletivo do esmalte marginal.

É importante frisar que, mesmo sob anestesia, a paciente queixou-se de sensibilidade no transoperatório, quando da remoção do tecido cariado, em ambas as restaurações.

Nas consultas seguintes, foram realizadas restauração em resina composta do 54 e exodontia do 75, além da instalação da banda-alça até a erupção do sucessor permanente.

Foi implementado um regime de consultas periódicas mensal durante 10 meses, a fim de acompanhar clinicamente o comportamento das restaurações, avaliando presença de possíveis falhas e suas razões, como fratura total ou parcial, cárie secundária, desgaste severo expondo dentina, dor, entre outros motivos. Com a remoção do aparelho banda-alça após 10 meses, em decorrência da erupção do dente permanente relativo ao espaço em manutenção, a criança teve seus tratamentos curativo e ortodôntico finalizados, sendo inserida num controle semestral de avaliação do tratamento realizado.

Após 16 meses das restaurações dos molares afetados pela HMI, observou-se que não houve perda de material restaurador em ambas as restaurações, havendo apenas um pequeno desgaste da restauração na região da cúspide mésio-vestibular do 
elemento 46 (Figura 4). A criança não relatou sensibilidade dentinária em estímulos térmicos e mecânicos e nem sintomatologia dolorosa. Constatou-se que houve uma melhora substancial no controle de placa. Os responsáveis foram informados sobre o desgaste da restauração do dente 46 e alertados quanto à importância das consultas de retorno para a melhor proservação do caso.

Figura 4 - Vista oclusal inferior referente ao tratamento restaurador dos dentes 36 e 46 após 16 meses de acompanhamento

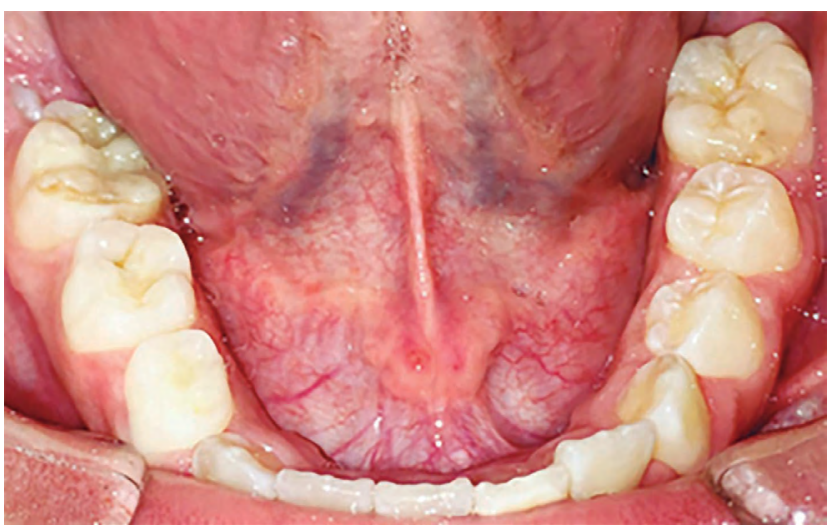

Fonte: autores.

\section{Discussão}

A tomada de decisão no tratamento de molares afetados severamente pela HMI é complexa, depende de vários fatores e necessita de um planejamento adequado para o paciente, visando, principalmente, à redução da sintomatologia dolorosa e à devolução funcional, estética e social.

No caso relatado, observou-se grande perda de estrutura dentária, típica de fratura por força mecânica, com acúmulo de biofilme, pois a sensibilidade dificultava a higiene da criança. A exposição de dentina, a presença de biofilme e uma dieta rica em açúcar oportunizaram o desenvolvimento de cárie dentária e contribuíram para o aumento da sensibilidade, tanto provocada quanto espontânea ${ }^{6}$, principal queixa da paciente. Uma segunda hipótese para explicar o desenvolvimento de cárie dentária em dentes com HMI, proposta por Americano et al. ${ }^{14}$ (2017), é que a superfície do esmalte hipomineralizado é mais susceptível à adesão de bactérias, mesmo aparentando estar intacta, uma vez que o tamanho dos poros são grandes o suficiente para permitir a invasão e a destruição de esmalte e dentina por bactérias cariogênicas. Diante dessas considerações, o presente caso foi classificado como severo, como proposto pela Academia Europeia de Odontopediatria ${ }^{15}$.

A literatura sugere uma variedade de opções de tratamento para molares afetados severamente pela $\mathrm{HMI}^{12,16}$. O tratamento adotado neste relato foi a restauração dos elementos pela técnica direta, utilizando a resina composta como material restaurador. A eleição desse material é justificada pela facilidade de realização de futuros reparos sem maiores perdas de estrutura, fornecendo suporte e proteção ao dente, além de minimizar o risco de falhas marginais e ter boa resistência ao desgaste $\mathrm{e}^{13,16}$. Uma revisão sistemática observou que a resina composta apresentou uma menor taxa anual de falha em molares hipomineralizados, quando comparada a cimento de ionômero de vidro, amálgama, compômeros e selantes de fossas e fissuras ${ }^{12}$. Ainda, os materiais adesivos são prioritariamente recomendados, uma vez que a necessidade de retenções mecânicas em um dente hipomineralizado não é indicada, assim, o uso do amálgama não tem sido incentivado ${ }^{12}$.

$\mathrm{O}$ cimento de ionômero de vidro é considerado um material isolante, liberador de flúor e que favorece a remineralização do tecido ${ }^{16}$, sendo bastante utilizado nos casos de HMI ${ }^{17,18}$. Porém, sua baixa resistência mecânica impede que seja usado como restaurador definitivo em molares afetados ${ }^{16} \mathrm{e}$, por isso, ele tem sido indicado como material provisório em pacientes com pouca maturidade, até que se possa realizar um tratamento mais complexo ${ }^{19}$. O seu uso como material de forramento em um dos dentes da paciente deste caso foi justificado pela cavidade apresentar-se próxima da câmara pulpar e causar grande sensibilidade dentinária. O CIV inserido na cavidade diminui a quantidade de compósito e, por conseguinte, há redução da contração de polimerização, reduzindo a sensibilidade ${ }^{18,20}$.

Um ensaio clínico randomizado de 18 meses de acompanhamento não observou diferença entre o uso de adesivo autocondicionante e o de adesivo convencional no que concerne à sobrevivência clínica de restaurações em resina composta em primeiros molares permanentes afetados pela $\mathrm{HMI}^{21}$, respaldando a utilização dos diferentes tipos de adesivo para cada dente neste relato de caso. Embora os adesivos autocondicionantes dispensem o condicionamento ácido prévio, alguns autores têm recomendado um condicionamento seletivo das margens do esmalte $^{22,23}$, a fim de melhorar a adesão a este substrato. Por esta razão, esta técnica seletiva de condicionamento foi realizada no dente que recebeu o adesivo autocondicionante.

Outra alternativa de tratamento apontada pela literatura é a instalação de coroa metálica ${ }^{16}$, apresentando como vantagens a contenção da deterioração dentária, o controle da sensibilidade, o estabelecimento de um bom contato interproximal e a facilidade da técnica. Entretanto, se não estiver adequadamente adaptada, pode levar o paciente a problemas ortodônticos e periodontais ${ }^{13}$. É discutível ainda a indicação de coroas metálicas em crianças em desenvolvimento, uma vez que pode alterar a oclusão, sendo mais seguramente indicadas em dentição permanente ${ }^{15}$.

A exodontia do molar severamente hipomineralizado é outra possibilidade terapêutica, principalmente quando outros tratamentos menos invasivos 
falham ou há pouco remanescente dentário. Nesse caso, tornam-se necessários uma avaliação ortodôntica prévia e um posterior manejo do espaço ${ }^{12,13}$.

A hiperalgia relatada durante o transoperatório, mesmo sob anestesia local, já foi relatado em outro estudo ${ }^{24}$ e é provavelmente ocasionada por uma inflamação subclínica das células pulpares devido a uma maior porosidade do esmalte ${ }^{25}$ ou ainda por alterações nos canais de sódio decorrentes da inflamação tecidual ${ }^{26}$. Essa sensibilidade aumentada tem como consequência crianças mais propensas ao medo e à ansiedade ante o tratamento ${ }^{2}$. Por esse motivo, destaca-se a importância da agilidade e da habilidade do operador no manejo desses molares em paciente infantil. No caso da criança deste relato, quando da queixa de dor durante a remoção do tecido cariado, foi realizada complementação da anestesia do nervo alveolar inferior, com maior agilidade no transoperatório e condicionamento do comportamento da criança.

A literatura tem apontado que a HMI causa um maior impacto na qualidade de vida de crianças afetadas, seja pela dor, pela sensibilidade a estímulos térmicos ou pela aparência estética das opacidades nos incisivos ${ }^{7}$. Não houve relato de queixa estética das opacidades nos dentes anteriores pela criança deste relato de caso, sendo, por esse motivo, realizada apenas proservação das manchas. Para o mascaramento dessas lesões, a literatura não é conclusi$\mathrm{va}^{12}$, entretanto, terapias remineralizadoras, microabrasão e facetas diretas e indiretas são condutas propostas $^{27}$.

Em 16 meses de acompanhamento, não foi observada perda de material e nem falhas que comprometessem a função. A longevidade das restaurações em dentes posteriores vai muito além dos materiais e da técnica empregada, fatores como tipo e localização do dente, qualidade técnica do operador, elementos socioeconômicos, demográficos e comportamentais devem ser levados em consideração ${ }^{28}$. O desgaste observado em uma cúspide de um dos dentes merece um acompanhamento periódico, a fim de observar evolução ou não.

Durante as consultas de acompanhamento, a criança apresentou uma melhora na higiene bucal e um maior interesse no autocuidado, assim como por parte do cuidador. As queixas de dor e sensibilidade não foram mais relatadas, não justificando, pois, procedimentos para sensibilidade, diferentemente de Assunção et al. ${ }^{29}$ (2014), em que, embora o diagnóstico assemelhe-se ao caso clínico deste estudo, houve a necessidade de reduzir a sensibilidade após o procedimento restaurador, por meio de aplicação tópica diária de flúor gel neutro.

Há uma forte relação entre presença de hipomineralizações nos segundos molares decíduos e a presença desta mesma condição nos primeiros molares permanentes ${ }^{30,31}$. Assim sendo, o diagnóstico precoce das opacidades no elemento 85 poderia ter servido de sinalizador, permitindo um acompanhamento enfático da erupção dos molares permanentes, aumentando as chances de evitar o agravamento da perda de estrutura dentária.

Conhecer as possíveis causas das patologias do esmalte torna-se importante para o clínico, uma vez que, além de auxiliar no diagnóstico diferencial, frequentemente, pais ou responsáveis procuram o cirurgião-dentista com questões sobre os motivos que desencadearam as lesões no esmalte, como foi o caso deste relato. Ainda que a etiologia da HMI seja considerada inconclusiva ${ }^{32}$, as doenças da infância, particularmente as febris, parecem ter uma considerável associação com a $\mathrm{HMI}^{33}$.

Quando realizada a anamnese da criança deste relato, não se percebeu alteração nos períodos pré-natal e perinatal, porém foram relatados repetitivos quadros de infecções do trato urinário, acompanhados de febre alta e, por conseguinte, uso frequente de antibióticos. Esses fatores podem estar envolvidos na etiologia da HMI, uma vez que a época dessa condição sistêmica coincidiu com o período de maturação da matriz orgânica do esmalte dos primeiros molares e incisivos permanentes ${ }^{34}$. O mecanismo que explica a associação de quadros febris com a HMI é a possibilidade de a alta temperatura poder gerar uma disfunção ameloblástica e até uma degeneração celular completa ${ }^{35}$. Entretanto, por se tratar de uma doença multifatorial e com susceptibilidade genética, outros fatores etiológicos não devem ser desconsiderados ${ }^{32}$.

\section{Considerações finais}

É importante que o cirurgião-dentista saiba diferenciar a HMI dos demais DDE, bem como avaliar o grau de severidade dos dentes afetados, a fim de eleger a melhor conduta. Além disso, é relevante o monitoramento clínico, uma vez que não se sabe ainda a sobrevivência das restaurações diretas nestes dentes. O tratamento proposto para o caso mostrou-se satisfatório durante o período avaliado, pois recuperou a funcionalidade dos dentes afetados pela HMI e não houve mais relatos de sintomatologia dolorosa. A paciente será mantida em acompanhamento para avaliação da proposta terapêutica e do comprometimento estético dos dentes anteriores.

\section{Abstract}

Objective: to report a clinical case of a child diagnosed with severe Molar-Incisor Hypomineralization (MIH) and to present the therapeutic proposal and follow-up. Case report: female patient, six years old, referred to a dental service with the major complaint of "pain in the lower tooth (right side)". The clinical examination showed demarcated opacities on the dental enamel ranging from white to brownish shades, typical of hypomineralization, on the buccal surfaces of teeth 11, 12, 21, 32, 31 , and 41 and occlusal surfaces of teeth 16 and 26, without loss of dental structure. Teeth 36 and 46 showed, 
in addition to the demarcated opacities, post-eruptive enamel fractures associated with active dentin carious lesions. Clinically and radiographically, there were no signs of compromise to pulp health. The child was diagnosed with $\mathrm{MIH}$. The treatment consisted of direct resin composite restorations of elements 36 and 46 . After 16 months, no loss of restorative material was observed in both restorations. The child reported neither dentin sensitivity nor painful symptomatology. Final considerations: the treatment proposed for the case was satisfactory during the time evaluated, recovering the functionality of the teeth affected by the MIH without showing further episodes of sensitivity and pain.

Keywords: Dental care. Child. Tooth demineralization. Dental enamel. Oral health.

\section{Referências}

1. Koch G, Hallonsten AL, Ludvigsson N, Hansson BO, Holst A, Ullbro C. Epidemiologic study of idiopatic enamel hypomineralization in permanent teeth of Swedish children. Comm Dent Oral Epidemiol 1987; 15(4):279-85.

2. Jälevik B, Klingberg GA. Dental treatment fear and behaviour management problems in children with severe enamel hypomineralization of their permanent first molars. Int $\mathrm{J}$ Pediatr Dent 2002; 12(1):24-32.

3. Leppaniemi A, Lukinmaa PL, Alaluusua S. Nonfluoride hypomineralizations in the permanent first molars and their impact on the treatment need. Caries Res 2001; 35(1):36-40.

4. Neville BW, Damm DD, White DK. Oral maxillo facial pathology. 3. ed. Rio de Janeiro: Elsevier; 2009.

5. Garg N, Jain AK, Saha S, Singh J. Essentiality of early diagnosis of molar incisor hypomineralization in children of its clinical presentation, etiology and management. Int J Child Pediatr Dent 2012; 5(3):190-6.

6. Dantas-Neta NB, Moura LF, Cruz PF, Moura MS, Paiva SM, Martins CC, et al. Impact of incisor-molar hypomineralization on oral health-related quality of life in school children. Braz Oral Res 2016; 30(1):e117.

7. Weerheijm KL, Jälewik B, Alaluusua S. Molar incisor hypomineralization. Caries Res 2001; 35(5):390-1.

8. Lygidakis NA, Dimou G, Marinou D. Molar-incisor-hypomineralisation (MIH). A retrospective clinical study in Greek children. II. Possible medical aetiological factors. Eur Arch Paediatric Dent 2008; 9(4):207-17.

9. Zhao D, Dong B, Yu D, Ren Q, Sun Y. The prevalence of molar incisor hypomineralization: evidence from 70 studies. Int J Paediatr Dent 2018; 28(2):170-9.

10. da Silva-Júnior IF, Aguiar NL, Barros WRC, da Silva LS, Arantes DC, Nascimento LS. Prevalence and severity of molar-incisor hypomineralization in students of Belém, Brazil. Braz Res Pediatric Dent Integ Clin 2015; 15(5):377-85.

11. Soviero V, Haubek D, Trindade C, Da Matta T, Poulsen S. Prevalence and distribution of demarcated opacities and their sequelae in permanent teeth $1^{\text {st }}$ molars and incisors in 7 to 13-years-old Brazilian children. Acta Odontol Scand 2009; 67(3):170-5.

12. Elhennawy K, Schwendicke FPD. Managing molar-incisor hypomineralization: a systematic review. J Dent 2016; 55:1624.

13. William V, Messer LB, Burrow MF. Molar-incisor hypomineralization: review and recommendations for a clinical management. Pediatric Dent 2006; 28(3):224-32.
14. Americano GC, Jacobsen PE, Soviero VM, Haubek D. A systematic review on the association between molar-incisor hypomineralization and dental caries. Int J Paediatric 2017; 27(1):11-21.

15. Lygidakis NA, Wong F, Jälevik B, Vierrou A-M, Alaluusua S, Espelid I. Best clinical practice guidance for clinicians dealing with children presenting with molar-incisor-hypomineralisation (MIH): an EAPD policy document. Eur Arch Paediatr Dent 2010; 11(2):75-81.

16. Fayle SA. Molar-incisor hypomineralization restorative management. Eur J Paediatric Dent 2003; 22(4):121-6.

17. Vilani PNL, Paim AS, Penido CVSR, Barra SG. Hipomineralização molar-incisivo: relato de caso clínico. Fac Odontol de Lins/Unimep 2014; 24(1):64-8.

18. Côrtes LCA, Floriano I, Tedesco TK, Imparato JCP. Protocolo de tratamento de hipomineralização molar-incisivo em odontopediatria: relato de caso clínico. J Bio Dent Biomater 2015; $5(2): 21-9$.

19. Fragelli CM, Souza JF, Jeremias F, Cordeiro R de C, SantosPinto L. Molar-incisor hypomineralization (MIH) conservative treatment management to restore affected teeth. Braz Oral Res 2015; 29(1):1-7.

20. Vieira IM, Louro RL, Atta MT, Navarro MFL, Francisconi PAS. O cimento de ionômero de vidro na Odontologia. Rev Saúde Com 2006; 2(1):75-84.

21. de Souza JF, Fragelli CB, Jeremias F, Paschoal MAB, Santos-Pinto L, de Cássia Loiola Cordeiro R. Eighteen-month clinical performance of composite resin restorations with different adhesive systems for molars affected by molar-incisor hypomineralization. Clin Oral Invest 2016; 21(5):1725-33.

22. Lopes LS, Malaquias P, Calazans FS, Reis A, Loguércio AD, Barceleiro MO. Protocolo das possibilidades técnicas de aplicação dos sistemas adesivos universais: revisão da literatura com relato de caso. Rev Bras Odontol 2016; 73(2):173-7.

23. Van Landuyt KL, Peumans M, De Munck J, Lambrechts P, Van Meerbeek B. Extension of a one-step self-etch adhesive into a multi-step adhesive. Dent Mater 2006; 22(6):533-44.

24. da Silva-Júnior IF. Tratamento de molares afetados pela hipomineralização molar-incisivo: uma revisão ilustrada da literatura [Trabalho de conclusão de curso de especialização]. Porto Alegre: Universidade Federal do Rio Grande do Sul; 2015.

25. Crombie FA, Manton DJ, Palamara JE, Zalizniak I, Cochrane NJ, Reynolds EC. Characteristics of developmentally hypomineralized human enamel. J Dent 2013; 41(10):611-8.

26. Rodd HD, Boissonade FM, Day PF. Pulpal status of hypomineralization of permanent molars. Pediatric Dent 2007; 29(6):514-20.

27. Wallace A, Deery C. Management of opacities in children and adolescents. Dent Update 2015; 42(12):951-8.

28. Demarco FF, Corrêa MB, Cenci MS, Moraes RR, Opdam NJ. Longevity of posterior composite restorations. Not only a matter of materials. J Dent 2012; 28(1):87-101.

29. Assunção CM, Girelli V, Sarti CS, Ferreira ES, Araujo FB, Rodrigues JA. Hipomineralização de molar-incisivo (HMI): relato de caso e acompanhamento tratamento restaurador. Rev Assoc Paul Cir Dent 2014; 68(4):346-50.

30. Aine L, Backström MC, Mäki R, Kuusela AL, Koivisto AM, Ikonen RS, et al. Enamel defects in primary and permanent teeth in children born prematurely . J Oral Pathol Oral Med 2000; 29(7):403-9.

31. Elfrink ME, ten Cate JM, Jaddoe VW, Hofman A, Moll HA, Veerkamp JS. Deciduous molar hypomineralization and molar-incisor hypomineralization. J Dent Res 2012; 91(6):551-5. 
32. Alallusua S. Aetiology of molar-incisor hypomineralization: a systematic review. Eur Arch Paediatric 2010; 11(1):53-8.

33. Silva MJ, Scurrah KJ, Craig JM, Manton DJ, Kilpatrick N. Etiology of molar-incisor hypomineralization: a systematic review. Comm Dent Oral Epidemiol 2016; 44(4):342-53.

34. Nanci A. Ten Cate's Oral histology: developement, structure and function. 8. ed. Rio de Janeiro: Elsevier; 2008.

35. Kreshover SJ, Clough OW, Bear DM. Prenatal influences on tooth development. I. Alloxan diabetes in rats. J Dent Res 1953; 32(2):246-61.

Endereço para correspondência:

Ivam Freire da Silva Júnior

Rua Gonçalves Chaves, 457, Centro

96015-560 - Pelotas, RS, Brasil

E-mail: ivamfreire@gmail.com

Recebido: 28/05/18. Aceito: 15/06/18. 